\title{
Franck-Hertz effect in cathodo- and photoluminescence of wide-gap materials
}

\author{
Aleksandr Lushchik*, Cheslav Lushchik, Tiit Kärner, Peeter Liblik, Vitali Nagirnyi, Evgeni Shablonin, \\ Anna Shugai, Evgeni Vasil'chenko \\ Institute of Physics, University of Tartu, Riia 142, Tartu 51014, Estonia
}

\section{A R T I C L E I N F O}

\section{Article history:}

Received 7 August 2009

Accepted 29 January 2010

\section{Keywords:}

Photo- and cathodoluminescence

Energy transfer by hot carriers

Wide-gap inorganic dielectrics

\begin{abstract}
A B S T R A C T
A brief overview of previously obtained and novel data on the manifestations of an analogue of FranckHertz effect in photo- and cathodoluminescence of wide-gap inorganic materials is presented. On the example of $\mathrm{NaCl}: \mathrm{Tl}^{+}$and $\mathrm{MgO}: \mathrm{Cr}^{3+}$ single crystals, the excitation processes of the luminescence of $6 s^{2} \mathrm{Tl}^{+}$ ions and $3 d^{3} \mathrm{Cr}^{3+}$ ions by $5-15 \mathrm{keV}$ electrons or $5-20 \mathrm{eV}$ photons at $6-420 \mathrm{~K}$ have been studied. The rapid processes of the direct energy transfer to $\mathrm{Tl}^{+}$by hot conduction electrons or to $\mathrm{Cr}^{3+}$ centers by hot electrons and/or hot valence holes have been separated from rapid excitonic and more inertial electronhole processes.
\end{abstract}

(c) 2010 Elsevier Ltd. All rights reserved.

\section{Introduction}

The main goal of the present paper is to sum up long-standing investigations of the solid-state analogue of Franck-Hertz effect (FHE) in gases - a resonant excitation of luminescent impurity centers and structural defects by hot (non-relaxed) conduction electrons or/and valence holes in wide-gap materials (WGM, $E_{\mathrm{g}}>6 \mathrm{eV}$ ) - performed at the Institute of Physics, Tartu. In such materials, the separation of FHE in low-temperature photo- and cathodoluminescence (PL and $\mathrm{CL}$, respectively) is of obvious interest for both fundamental studies and various technical applications, such as the elaboration of novel spectral transformers for neon luminescent tubes and xenon plasma display panels or the increase of WGM radiation resistance by weakening hot electronhole $(e-h)$ recombination with the creation of Frenkel defects (Lushchik et al., 2006).

In 1914, James Franck and Gustav Hertz (Nobel Prize in 1925) performed the now-famous experiment on the interaction of slow electrons of various kinetic energies with a mercury vapor and detected an ultraviolet emission $(4.9 \mathrm{eV})$ of free $\mathrm{Hg}$ atoms resonant to electron energy losses. Niels Bohr repeatedly cited this experiment as the most illustrative conformation of quantized model of the atom, when atoms absorb and emit discrete amounts of energy. From the modern standpoint, the resonant behavior of the excitation function of free mercury atoms by electrons of $\sim 4.9 \mathrm{eV}$ corresponds to spin-restricted singlet-triplet ${ }^{1} S_{0} \rightarrow{ }^{3} P_{1}$ electron transitions caused by an effective electron exchange. In the case of

\footnotetext{
* Corresponding author. Tel.: +372 7374619; fax: +372 7383033

E-mail address: aleksandr.lushchik@ut.ee (A. Lushchik).
}

totally allowed ${ }^{1} S_{0} \rightarrow{ }^{1} P_{1}$ electron transitions of free $\mathrm{Hg}$ atoms the excitation function smoothly increases with the rise of the kinetic energy of incident electrons above the threshold value.

At the beginning of 1960s, William Schockley and Viktor Vavilov separated an elementary act of multiplication of $e-h$ pairs in narrow-gap semiconductors. Thereafter, PL with quantum yield $\mathrm{QY}>1$ was obtained in alkali iodides doped with mercury-like $\mathrm{Tl}^{+}$ and $\mathrm{In}^{+}$ions (Ilmas et al., 1965). The exciting photon of vacuum ultraviolet (VUV) radiation was transformed into two or three quanta of visible emission of impurity centers. This so-called "photon multiplication" phenomenon was thoroughly studied at the Institute of Physics (Tartu, Estonia) in a number of alkali halide crystals (AHC), pure and doped with $s^{2}$ - and $d^{10}$-impurity ions. The analysis of the obtained luminescence data as well as of literature data on photoelectron spectra of AHC thin films allowed revealing two main mechanisms of photon multiplication connected with the creation of several electronic excitations by one exciting photon. A hot conduction electron with a sufficient energy, formed at the exciting photon absorption, is able to create a secondary exciton or a secondary $e-h$ pair. The subsequent radiative exciton decay and the recombination of a secondary and a primary cold $e-h$ pairs cause the appearance of two emission quanta per one exciting photon.

The application of synchrotron radiation allowed for a further detailed study of the excitonic and $e-h$ multiplication mechanisms in AHC (Ivanov et al., 1973; Lushchik et al., 1996; Feldbach et al., 2000). The elementary mechanisms of photon multiplication have been studied in binary and complex metal oxides as well (Lushchik et al., 2005, 2006). The separation and elucidation of the third multiplication mechanism in WGM - the solid-state analogue of FHE appeared to be much more complicated (Lushchik et al., 1966, 
1998, 2005; Feldbach et al., 1997; Kirm et al., 1997; Makhov et al., 2008). The process of the direct excitation of impurity centers by hot conduction electrons or valence holes is rather fast (nanoseconds) and hardly distinguishable from the low-inertial excitonic mechanism. To account for the mechanism of electroluminescence in wide-gap semiconductors of $\mathrm{ZnS}: \mathrm{Cu}^{+}$and $\mathrm{ZnS}: \mathrm{Mn}^{2+}$, the impact excitation of luminescent impurities by conduction electrons accelerated in an electric field was suggested (Piper and Williams, 1952; Bhattacharyya et al., 1993).

A brief consideration of experimental manifestations of FHE in PL of WGM, taking $\mathrm{NaCl}$ single crystals as an example, will be given in Section 2. Section 3 contains data on low-temperature CL of MgO single crystals in support of a solid-state analogue of FHE in metal oxides.

\section{Manifestations of FHE in photoluminescence of WGM}

Fig. 1 shows a simplified energy-band diagram of a typical WGM - a NaCl single crystal doped with mercury-like $\mathrm{Tl}^{+}$ions with a $6 s^{2}$ ground-state configuration. The minimum energy for the direct optical formation of an anion exciton with $n=1$ in $\mathrm{NaCl}$ equals $E_{\mathrm{e}}=8 \mathrm{eV}$, the energy gap is $E_{\mathrm{g}}=8.75 \mathrm{eV}$ and the total width of the valence band (v-band) is $E_{\mathrm{v}} \approx 3 \mathrm{eV}$. Fig. 1 also demonstrates the energy levels of a $\mathrm{Tl}^{+}$impurity center in $\mathrm{NaCl}$ :Tl, that are located within the energy gap and correspond to ${ }^{1} S_{0},{ }^{3} P_{1}$ and ${ }^{1} P_{1}$ states of a free $\mathrm{Tl}^{+}$ion. The energetic position of the ${ }^{1} P_{1}$ level $(\sim 6.2 \mathrm{eV})$ with respect to the bottom of a conduction band (c-band) is experimentally determined by measuring the excitation spectra of recombination luminescence and photoconductivity.

Fig. 2 demonstrates the excitation spectra of the A emission (4.27 eV, ${ }^{3} P_{1} \rightarrow{ }^{1} S_{0}$ ) of $\mathrm{Tl}^{+}$centers in $\mathrm{NaCl}: \mathrm{Tl}^{+}$measured using synchrotron radiation at $8 \mathrm{~K}$ (SUPERLUMI station of HASYLAB at DESY, Hamburg). The spectra were measured for time-integrated luminescence as well as for the emission detected within two time windows (length $\Delta t$ ) correlated with the excitation pulses of synchrotron radiations (delayed by $\delta t$ ). The parameters for the fast emission component were $\Delta t=10 \mathrm{~ns}$ and $\delta t=2 \mathrm{~ns}$ and for the slow one $-\Delta t=250 \mathrm{~ns}$ and $\delta t=200 \mathrm{~ns}$. The spectra contain three bands:

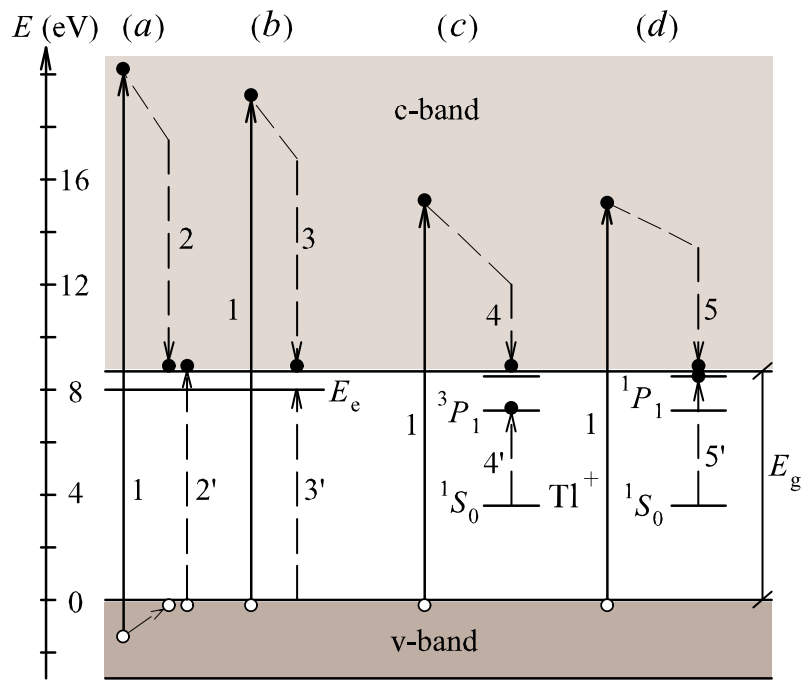

Fig. 1. A simplified energy-band diagram for a $\mathrm{NaCl}: \mathrm{Tl}^{+}$crystal. The multiplication processes connected with the creation of a secondary $e-h$ pair (case $a$ ) or a secondary anion exciton (case $b$ ) by a hot conduction electron as well as the excitation of a $\mathrm{Tl}^{+}$ impurity center by a hot electron (cases $c$ and $d$ ) due to non-radiative Auger transitions $\left(2 \rightarrow 2^{\prime}, 3 \rightarrow 3^{\prime}, 4 \rightarrow 4^{\prime}, 5 \rightarrow 5^{\prime}\right)$. All the processes start with the absorption of an exciting photon (arrow line 1). The inclined dashed arrow lines demonstrate nonradiative transitions at carrier relaxation inside energy bands.

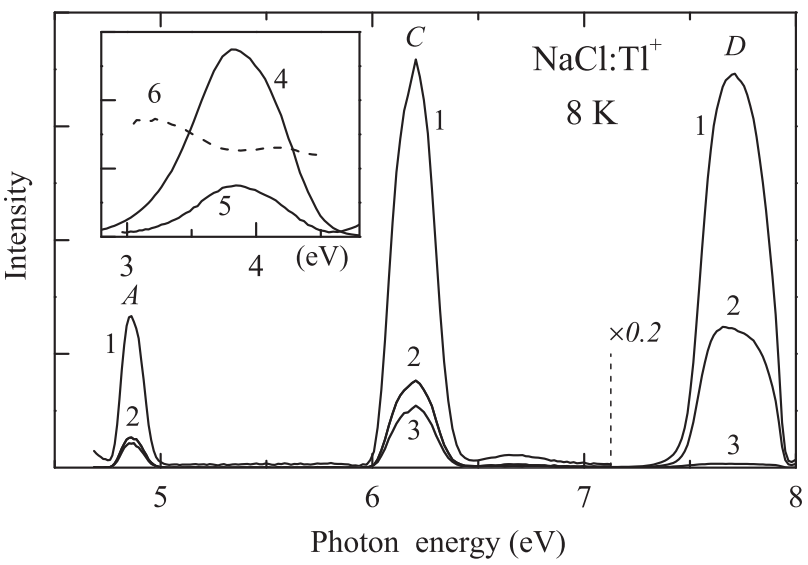

Fig. 2. The excitation spectra of $\mathrm{A}$ emission $\left(4.27 \mathrm{eV},{ }^{3} P_{1} \rightarrow{ }^{1} S_{0}\right)$ of $\mathrm{Tl}^{+}$centers measured for time-integrated (curve 1 ), slow ( $\Delta t=250 \mathrm{~ns}$ and $\delta t=200 \mathrm{~ns}$, curve 2 ) and fast $\left(\Delta t=10 \mathrm{~ns}\right.$ and $\delta t=2 \mathrm{~ns}$, curve 3 ) components in $\mathrm{NaCl} \mathrm{Tl}^{+}$at $8 \mathrm{~K}$. Inset shows the emission spectra measured for slow (4) and fast (5) components and the intensity ratio spectrum for slow/fast emissions (6) in $\mathrm{NaCl}$ on the excitation by $7.7 \mathrm{eV}$ photons at $8 \mathrm{~K}$.

the A excitation (absorption) band peaked at $4.78 \mathrm{eV}$ corresponds to ${ }^{1} S_{0} \rightarrow{ }^{3} P_{1}$ electron transitions in a free $\mathrm{Tl}^{+}$ion; the C-band $(6.2 \mathrm{eV})$ is related to ${ }^{1} S_{0} \rightarrow{ }^{1} P_{1}$ singlet-singlet transitions and the so-called D-band (a non-elementary band at $\sim 7.7 \mathrm{eV}$ directly adjoins the exciton absorption bands), which is caused by the excitation and ionization of the chlorine ions surrounding the impurity ion.

According to Fig. 1, a photon of $h v>20 \mathrm{eV}$ forms a hot conduction electron which is able to create a secondary $e-h$ pair due to the non-radiative Auger transition (case $a$, arrow lines 2 and $2^{\prime}$ ), while the energy excess of a hot conduction electron (with respect to the bottom of the c-band), formed by an exciting photon of $h \nu=18-$ $20 \mathrm{eV}$ is sufficient to create a secondary exciton (case $b, 3$ and $3^{\prime}$ ). Even a lower energy of exciting photons ( $h \nu=15-17 \mathrm{eV})$ is needed for the direct excitation of impurity centers by hot conduction electrons, i.e., for the realization of a solid-state analogue of FHE in $\mathrm{NaCl}: \mathrm{Tl}^{+}$(case $c$, arrow lines 4 and $4^{\prime}$ or case $d, 5$ and $5^{\prime}$ ).

A comparison of the excitation spectra of impurity center luminescence in doped crystals with the photoemission spectra from thin films had a beneficial effect on the separation of FHE from other mechanisms of photon multiplication in WGM. Earlier, this comparison had been made for $\mathrm{RbCl}: \mathrm{Tl}^{+}$and $\mathrm{RbCl}: \mathrm{Ag}^{+}$crystals (Feldbach et al., 1997; Lushchik et al., 1998). Fig. 3 shows the energy distribution curves of the emitted electrons on the excitation of an extra pure $\mathrm{NaCl}$ evaporated film by $14.7-17.5 \mathrm{eV}$ photons at $295 \mathrm{~K}$.

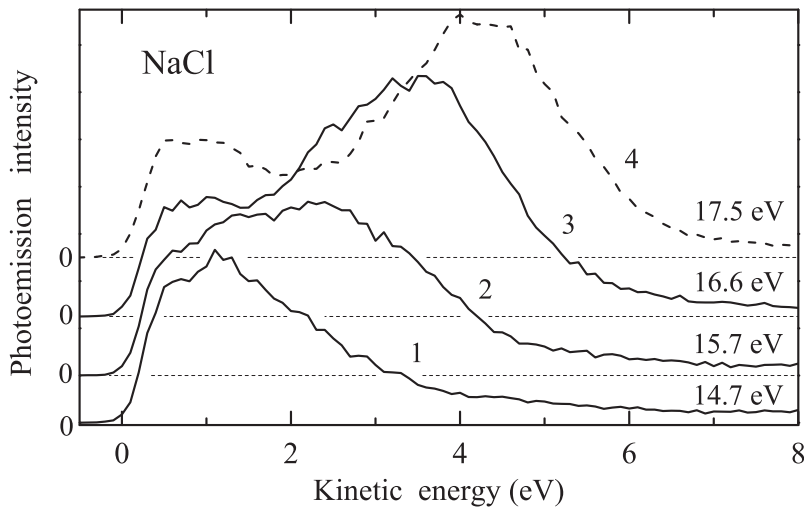

Fig. 3. Energy distribution curves of the emitted electrons of a $\mathrm{NaCl}$ thin film for various exciting photon energies at $295 \mathrm{~K}$. 
The photoelectron spectra were measured at beam-line $6 \mathrm{~A} 2$ at the UVSOR facility (Okazaki, Japan). The kinetic energy ( $\left.E_{\mathrm{kin}}\right)$ of the emitted electrons is given relative to the vacuum level, while the energy of the conduction electrons inside a crystal is higher by a value of an electron affinity $\chi=0.7 \mathrm{eV}$.

According to Fig. 3, photons of $h \nu=15.7 \mathrm{eV}$ already create the electrons with $E_{\text {kin }}=4.3 \mathrm{eV}$, which are able ( $\chi$ should be taken into account) to excite $\mathrm{Tl}^{+}$up to ${ }^{3} P_{1}$ state. A further radiative transition to a ground state $\left({ }^{3} P_{1} \rightarrow{ }^{1} S_{0}\right)$ results in the $A$ emission of $\mathrm{Tl}^{+}$ centers. In case $h \nu=16.6 \mathrm{eV}$, hot conduction electrons excite $\mathrm{Tl}^{+}$ centers even up to a higher ${ }^{1} P_{1}$ singlet state. The inset in Fig. 2 shows the emission spectra measured for the fast $(\Delta t=10 \mathrm{~ns}$, $\delta t=2 \mathrm{~ns})$ and slow ( $\Delta t=250 \mathrm{~ns}, \delta t=200 \mathrm{~ns})$ component in $\mathrm{NaCl}$ at the excitation by $7.7 \mathrm{eV}$ photons at $8 \mathrm{~K}$. Such exciting photons fall within a non-elementary $\mathrm{D}$ excitation band of $\mathrm{Tl}^{+}$centers adjacent to the exciton absorption. The intensity ratio spectrum for the slow and fast emission components makes it possible to separate the $\mathrm{A}$ emission of $\mathrm{Tl}^{+}$centers $(\sim 4.2 \mathrm{eV})$ and a more inertial emission $(\sim 3.35 \mathrm{eV})$ associated with self-trapped excitons.

By way of concluding this section, it should be mentioned that Fig. 1 presents a significantly simplified energy diagram of a $\mathrm{NaCl}$ crystal with a relatively narrow valence band $\left(E_{\mathrm{V}} \approx 3 \mathrm{eV}\right)$. The separation of FHE from the fast excitonic and $e-h$ mechanisms of photon multiplication in binary and complex metal oxides runs into obvious problems due to a sharp rise of $E_{\mathrm{v}}$ up to the value of $E_{\mathrm{g}}$ in these WGM. For instance, the value of $E_{\mathrm{v}} \approx 6.6 \mathrm{eV}$ can be experimentally estimated from the spectra of fast intraband luminescence measured under excitation of MgO crystals by nanosecond single $300 \mathrm{eV}$ electron pulses (Lushchik et al., 2006).

\section{Manifestations of FHE in cathodoluminescence}

Luminescent WGM have been successfully used for years as cathodoluminophors. The recombination of totally relaxed conduction electrons and valence holes mainly takes place at the final stage of the interaction of 50-5 keV electrons with WGM. The excitation of WGM by an electron beam was used over years at the Institute of Physics, Tartu, as a convenient way of investigating energy transfer processes to luminescent impurities - both mainly inertial $e-h$ processes and usually fast excitonic ones (see, e.g., Soovik and Eksina, 1964; Lushchik et al., 2005). The spectra of electron energy losses in WGM have been measured in many laboratories. These spectra demonstrated that, besides the creation of $e-h$ pairs and small-radius excitons, the energy is also spent on the so-called near-surface losses connected with the formation of electronic excitations, the energy of which is lower than that needed for bulk exciton creation $\left(E_{\mathrm{e}}\right)$. In the WGM with a high concentration of impurity ions or defects, these low-energy losses can be caused in part by FHE.

Recently, the spectra of steady CL were measured using a twochannel system with a double VUV (4-11 eV) and a double prism monochromator (1.8-6.0) at the excitation of pure and doped WGM (LiF, $\mathrm{NaCl}, \mathrm{MgO}, \mathrm{Al}_{2} \mathrm{O}_{3}, \mathrm{Y}_{2} \mathrm{O}_{3}, \mathrm{YAlO}_{3}, \mathrm{Lu}_{3} \mathrm{Al}_{5} \mathrm{O}_{12}, \mathrm{Gd}_{2} \mathrm{SiO}_{5}, \mathrm{CaSO}_{4}$, etc.) by an electron beam at 5-8 $\mathrm{K}$. The main experiments were performed with $5 \mathrm{keV}$ electrons ( $100 \mathrm{nA}, \sim 2 \mathrm{~mm}^{-2}$ spot). In the case of $15-20 \mathrm{keV}$ electrons, the possible charging of the irradiated single crystal was eliminated by the sputtering of a thin layer of $\mathrm{Au}$ $(\sim 10 \mathrm{~nm})$ on the sample surface. After the electron irradiation was stopped, it was possible to register the spectra of phosphorescence at a low temperature and the thermally stimulated luminescence (TSL) curves of the irradiated WGM with a heating rate of $\beta=10 \mathrm{~K} \mathrm{~min}^{-1}$.

It is worth noting that $1-2 \mathrm{~s}$ after the electron irradiation was stopped (the measurements of CL spectra usually take 10-25 min), the intensity of impurity or defect-related emission drastically decreased by 3-4 orders of magnitude, weakened by a factor of 10 within a minute and continued its slow damping. At a further crystal heating, the TSL was measured above a pedestal of tunnel phosphorescence that is practically independent of temperature in a wide range.

In the present paper, the manifestations of FHE in $\mathrm{CL}$ will be illustrated on the example of $\mathrm{MgO}$ and $\mathrm{MgO}: \mathrm{Cr}^{3+}$. The $\mathrm{MgO}$ single crystals were grown in our laboratory from a highly pure starting material by a variation of the arc fusion method. In $\mathrm{MgO}$, $E_{\mathrm{g}}=7.782 \mathrm{eV}$ and an exciton has a large radius and a low oscillator strength. The formation energy of this Vannier-Mott-type exciton with $n=1$ equals $E_{\mathrm{e}}=7.77 \mathrm{eV}$. The thermal quenching of exciton emission (nearly resonant with exciton absorption) occurs already at $80 \mathrm{~K}$ and all excitons undergo ionization at $80-300 \mathrm{~K}$. So far, there is no experimental evidence of the self-trapping of excitons, electrons and holes in the bulk of MgO crystals. Even at low temperatures $(T<80 \mathrm{~K})$, the creation efficiency of free excitons by $3-30 \mathrm{keV}$ electrons is at least an order of magnitude as low as of separated $e$ and $h$. Hence, mainly rapid $e-h$ recombination near/at impurity centers is in real competition with the fast energy transfer from an $\mathrm{MgO}$ matrix to impurities/defects via FHE.

The dependence of the $\mathrm{CL}$ intensity $\left(I_{\mathrm{CL}}\right)$ on temperature has been measured at the cooling down $(400 \rightarrow 6 \mathrm{~K})$ of $\mathrm{MgO}: \mathrm{Cr}^{3+}$. MgO crystals with a concentration of $\mathrm{Cr}^{3+}$ impurity ions of 0.01 at.\% were also grown in our laboratory. The absorption and emission of chromium centers in the region of $1.5-6.0 \mathrm{eV}$ have been earlier studied in detail for the same crystals (Kück et al., 2000). To avoid a high irradiation dose, short-run (30 s) pulses of an electron beam ( $5 \mathrm{keV}, 50 \mathrm{nA} \mathrm{mm}^{-2}$ ) and a subsequent $60 \mathrm{~s}$ pause were used at the cooling of $\mathrm{MgO}: \mathrm{Cr}^{3+}$ with $\beta=10 \mathrm{~K} \mathrm{~min}^{-1}$ (see Fig. 4). Only cooling below $45 \mathrm{~K}$ was performed under steady electron irradiation. The emission of $\mathrm{Cr}^{3+}$ ions $(\sim 1.76 \mathrm{eV})$ was selected through a double monochromator. In 1-2 s after a pulse of an electron irradiation was stopped, the intensity of CL drastically decreased. A repeated switch of an electron beam (except for the first time) caused a sharp jump

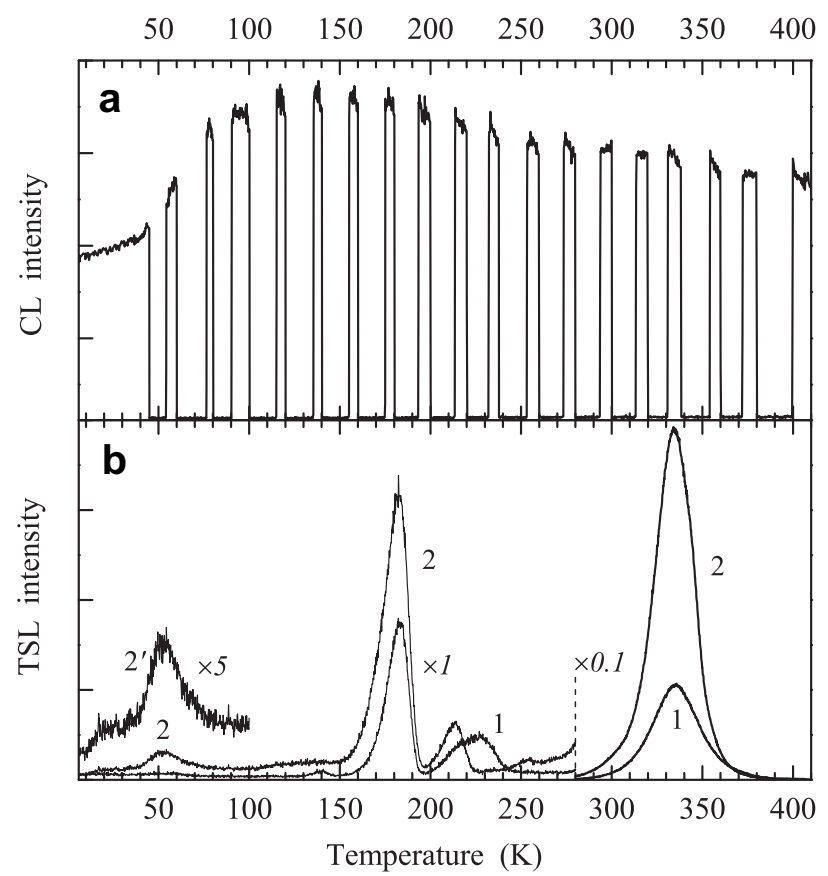

Fig. 4. a) The dependence of the $\mathrm{CL}$ intensity on the cooling temperature $(400 \mathrm{~K} \rightarrow$ $6 \mathrm{~K})$ for a $\mathrm{MgO}: \mathrm{Cr}^{3+}(0.01$ at. \%) crystal under excitation by $5 \mathrm{keV}$ electrons. (b) The TSL curves measured for $3.4 \mathrm{eV}$ (curve 1 ) and $3.2 \mathrm{eV}$ emission (curves 2 and $2^{\prime}$ ) in the $\mathrm{MgO}$ crystals irradiated by $15 \mathrm{keV}$ electrons at $6 \mathrm{~K}$. The samples were previously annealed at $1575 \mathrm{~K}(1)$ or plastically deformed $(\epsilon=4 \%)$ at $295 \mathrm{~K}\left(2,2^{\prime}\right) . \beta=10 \mathrm{~K} \mathrm{~min}^{-1}$. 
of a signal (not depicted in the figure) and the conversion thereof into a quasi-steady CL (this CL is shown) occurs in 1-2 s. According to Fig. $4 \mathrm{a}$, the intensity of $\mathrm{CL}$ (for a $\mathrm{Cr}^{3+}$-emission) slightly increases with a cooling of the sample down to $170 \mathrm{~K}$, remains nearly constant down to $115 \mathrm{~K}$ and significantly decreases down to about $55 \%$ from the maximum value at $80-40 \mathrm{~K}$. The same dependence of $I_{\mathrm{CL}}(T)$ can be obtained again after an additional preheating of the sample to $600 \mathrm{~K}$. A direct measuring of the pulse annealing of the EPR signal of paramagnetic centers in a $\mathrm{MgO}: \mathrm{Cr}^{3+}$ crystal irradiated at $80 \mathrm{~K}$ showed that the decay of the $\mathrm{Cr}^{1+}$ centers formed via electron trapping by $\mathrm{Cr}^{3+}$ occurs at $\sim 170 \mathrm{~K}$ (Kärner et al., 1980). However, the main part of $\mathrm{Cr}^{3+}$ centers can be restored only at $330-420 \mathrm{~K}$, when holes free themselves from $V^{0}, V_{\mathrm{OH}}$ and $\mathrm{V}^{-}$ centers and recombine with $\mathrm{Cr}^{2+}$ centers.

The results presented in Fig. 4a testify that a solid-state analogue of FHE provides in $\mathrm{MgO}: \mathrm{Cr}^{3+}$ a considerable contribution to a fast $\left(10^{-3}-10^{-6} \mathrm{~s}\right)$ component of $\mathrm{CL}$ associated with ${ }^{2} T_{1} \rightarrow{ }^{4} A_{2}$ and ${ }^{2} E \rightarrow{ }^{4} A_{2}$ radiative transitions in $\mathrm{Cr}^{3+}$ centers. This conclusion is especially apparent for the temperature region of 400-180 K, when the competition with the excitons ionized at such temperatures is excluded and $\mathrm{Cr}^{1+}$ centers are unstable. However, the situation is more complicated at low temperatures.

In order to influence drastically the $e-h$ recombination, the number of point and quasi-two-dimensional structural defects was significantly increased in the $\mathrm{MgO}$ crystals by a plastic deformation along [001] direction. Fig. 4b shows the TSL curves (measured for 3.4 and $3.2 \mathrm{eV}$ emissions) for two MgO crystals. The first crystal was annealed at $1575 \mathrm{~K}(2 \mathrm{~h})$ in an effort to anneal the dislocations and other defects while the second one was plastically deformed at $295 \mathrm{~K}$ (the thickness was decreased by $\epsilon=4 \%$ ). Plastic deformation causes a sharp enhancement of the TSL peaks at 335 and $50 \mathrm{~K}$. According to EPR studies, the $335 \mathrm{~K}$ peak is connected with the release of one of two holes from a $V^{0}$ center (two holes in a field of a cation vacancy, $v_{\mathrm{c}} h h$ ). The peak at $50 \mathrm{~K}$ is, in our opinion, associated with the electron release from shallow traps - bivacancies $\left(v_{\mathrm{a}} v_{\mathrm{c}}\right)$, the number of which is significantly increased in the deformed sample.

At low temperatures, the electron trapping at $v_{\mathrm{a}} v_{\mathrm{c}}$ is in competition with the direct excitation of $\mathrm{Cr}^{3+}$ centers by hot conduction electrons. As a result, the intensity of CL drastically decreases at the cooling of a deformed $\mathrm{MgO}$ crystal from 185 to $35 \mathrm{~K}$. The dependence of the $\mathrm{CL}$ intensity on the cooling temperature (the measurements were performed in the same way as for $\mathrm{MgO}: \mathrm{Cr}^{3+}$ see Fig. 4a) shows that $I_{\mathrm{CL}}$ decreases by a factor of 8 in the region of 180-35 $\mathrm{K}$ (for undeformed $\mathrm{MgO}: \mathrm{Cr}^{3+} \mathrm{I}_{\mathrm{CL}}$ at $35 \mathrm{~K}$ was only twice as low as at $180 \mathrm{~K}$ ). The defects formed in a deformed crystal serve as efficient centers of electron localization.

The excitation spectra for 2.9 and $5.2 \mathrm{eV}$ emissions in virgin and plastically deformed $(\epsilon=4 \%, T=295 \mathrm{~K}) \mathrm{MgO}$ crystals have been measured using synchrotron radiation at $8 \mathrm{~K}$. The efficiency of a broadband luminescence at $\sim 2.9 \mathrm{eV}$ sharply increases in the deformed sample in a wide spectral region of $h \nu=6.7-7.5 \mathrm{eV}$. Similar broad non-elementary excitation bands of luminescence connected with the absorption of anions surrounding $v_{\mathrm{a}} v_{\mathrm{c}}$ were revealed in many AHC (Lushchik et al., 1991). The emission of $5.2 \mathrm{eV}$ is effectively excited in a narrow region slightly below and above the exciton resonance. According to theoretical estimates (Seitz, 1954), the presence of $v_{c}$ causes the shift of neighbor anion absorption toward a short-wavelength region with respect to the absorption of anions in a regular lattice. It is worth noting that $\sim 5 \mathrm{eV}$ emission is well-expressed in $\mathrm{MgO}$ and $\mathrm{MgO}: \mathrm{Al}^{3+}$ crystals and is caused by the recombination of electrons with $V^{-}$and $V_{\mathrm{Al}}$ centers incorporating $v_{\mathrm{c}}$ (Kärner et al., 1980). The experiments with plastically deformed and preheated $\mathrm{MgO}$ crystals allow us to ascribe the low-temperature attenuation of the process of a fast ( $\mu \mathrm{s}$ scale) energy transfer to $\mathrm{Cr}^{3+}$ centers by hot electrons and holes to an interception of $e$ and $h$ by structural defects, the number of which can be decreased due to the preheating of a single crystal or can be increased by even a weak plastic deformation.

\section{Concluding remarks}

According to the photoelectron spectra and electron energy losses spectra measured by several authors, hot electrons, the energy of which is less than that needed for the creation of secondary excitons and $e-h$ pairs but is sufficient for the direct excitation of impurity ions and structural defects, are formed at the final stages of electronic excitation relaxation under irradiation of WGM by incident electrons or VUV radiation. In the WGM doped with various impurity ions, the manifestations of the direct energy transfer by hot conduction electrons and, tentatively, by hot valence holes to luminescent impurity centers were detected earlier in PL excited by VUV radiation of $h \nu=(1.5-4) E_{\mathrm{g}}$ or CL excited by $1-15 \mathrm{keV}$ electrons. In the present study, novel data on a solid-state analogue of FHE are provided on the example of $\mathrm{NaCl}: \mathrm{Tl}^{+}$and $\mathrm{MgO}: \mathrm{Cr}^{3+}$ single crystals. It is established that the creation energy of a pair of Frenkel $\left(E_{\mathrm{FD}}\right)$ defects is higher than $E_{\mathrm{g}}$ in $\mathrm{NaCl}$ at $T<100 \mathrm{~K}$ and in $\mathrm{MgO}$ in the whole investigated temperature region $(4-600 \mathrm{~K})$. This inequality $E_{\mathrm{FD}}>E_{\mathrm{g}}$ provides a high radiation resistance of these materials against non-impact mechanisms of radiation damage and promotes the investigation of FHE.

\section{Acknowledgements}

This work was supported by the Estonian Science Foundation (Grant 7825) and the European Community - Research Infrastructure Action under the FP6 "Structuring the European Research Area" Programme (through the Integrated Infrastructure Initiative IA-SFS).

\section{References}

Bhattacharyya, K., Goodnick, S.M., Wager, J.F., 1993. Monte Carlo simulation of electron transport in alternating-current thin-film electroluminescent devices. J. Appl. Phys. 73, 3390-3395.

Feldbach, E. Kamada, M., Kirm, M. Lushchik, A., Lushchik, C.H., Martinson, I., 1997. Direct excitation of $\mathrm{Tl}^{+}$impurity ions by hot photoelectrons in wide-gap crystals. Phys. Rev. B. 56, 13908-13915.

Feldbach, E., Kirm, M., Lushchik, A., Lushchik, C.H., Martinson, I., 2000. Excitonic and electron-hole processes in $\mathrm{NaCl}$ and $\mathrm{NaCl}: \mathrm{Ag}$ crystals under conditions of multiplication of electronic excitations. J. Phys. Condens. Matter 12, 1991-2005.

Ilmas, E.R., Liidja, G.G., Lushchik, C.H.B., 1965. Photon multiplication in crystals. I and II. Opt Spectrosc. (USA) 18, 255-259. and 359-361.

Ivanov, S.N., Ilmas, E.R., Lushchik, C.H.B., Mikhailin, V.V., 1973. Photon multiplication in $\mathrm{KCl}$ and $\mathrm{NaCl}$ crystals. Sov. Phys. Solid State (USA) 15, 1053-1055.

Kirm, M., Feldbach, E., Lushchik, A., Lushchik, C.H., Martinson, I., 1997. Excitation of $\mathrm{Tl}^{+}$centers by hot photoelectrons in alkali halide crystals. J. Lumin. 70, 939-941.

Kärner, T.N., Malysheva, A.F., Maaroos, A.A., Mürk, V.V., 1980. Thermally stimulated luminescence of MgO single crystals at temperatures 4.2-600 K. Sov. Phys. Solid State (USA) 22, 1178-1183.

Kück, S., Fornasiero, L., Heumann, E., Mix, E., Huber, G., Kärner, T., et al., 2000. Investigation of $\mathrm{Cr}$-doped $\mathrm{MgO}$ and $\mathrm{Sc}_{2} \mathrm{O}_{3}$ as potential laser sources for the near infrared spectral range. Laser Phys. 10, 411-416.

Lushchik, C.H.B., Ilmas, E.R., Savikhina, T.I., 1966. The mechanism of photon multiplication in crystals. In: Proceedings of the International Conference on Luminescence. Hungarian Acad. Sci., Budapest, pp. 99-104.

Lushchik, A., Lushchik, C.H., Lushchik, N., Frorip, A., Nikiforova, O., 1991. Formation and decay of electronic excitations localized near divacancies. Phys. Status Solidi. B 168, 413-423.

Lushchik, A., Feldbach, E., Kink, R., Lushchik, C.H., Kirm, M., Martinson, I., 1996. Secondary excitons in alkali halide crystals. Phys. Rev. B. 53, 5379-5387.

Lushchik, A., Kamada, M., Kirm, M., Lushchik, C.H., Martinson, I., 1998. Direct excitation of impurity centres by hot photoelectrons in ionic crystals. Radiat. Meas. 29, 229-334.

Lushchik, A., Lushchik, C.H., Feldbach, E., Kudrjavtseva, I., Liblik, P., Maaroos, A., Nagirnyi, V., Vasil'chenko, E., Savikhin, F., 2005. Photon multiplication in widegap BAM and SAM aluminates. Proc. SPIE 5946, 594609 
Lushchik, A., Lushchik, C.H., Kirm, M., Nagirnyi, V., Savikhin, F., Vasil'chenko, E., 2006. Defect creation caused by the decay of cation excitons and hot electron-hole recombination in wide-gap dielectrics. Nucl. Instrum. Methods B 250, 330-336. Makhov, V.N., Lushchik, A., Lushchik, C.H.B., Kirm, M., Vasil'chenko, E., Vielhauer, S. et al., 2008. Luminescence and radiation defects in electron-irradiated $\mathrm{Al}_{2} \mathrm{O}_{3}$ and $\mathrm{Al}_{2} \mathrm{O}_{3}: \mathrm{Cr}$. Nucl. Instrum. Methods B 266, 2949-2952.
Piper, W.W., Williams, F.E., 1952. Electroluminescence of single crystals of ZnS: Cu. Phys. Rev. 87, 151-152.

Seitz, F., 1954. Color centers in alkali halide crystals. Revs. Modern Phys. 26 7-94.

Soovik, T., Eksina, T., 1964. Kinetics of cathodoluminescence in alkali halide crystals. Trudy Inst. Fiz. AN Eston.SSR 26, 167-181 (in Russian). 